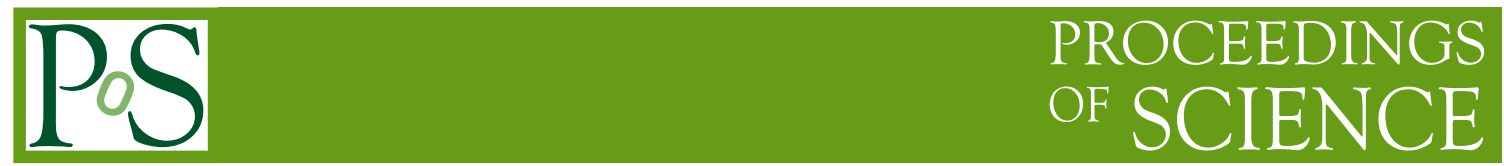

\title{
Crystal Based Double Beta Decay Experiments
}

\section{A. Garfagnini*i}

Università degli Studi di Padova and INFN

E-mail: alberto.garfagnini@unipd.it

The study of neutrinoless double beta decay (DBD) is the only presently known approach to the fundamental question if the neutrino is a Majorana particle, i.e. its own anti-particle. The observation of neutrinoless DBD would not only establish the Majorana nature of the neutrino but also allow to determine its effective mass if the nuclear matrix elements are given. The status of experiments under construction or in commissioning phase using semiconductor detectors technlogies is reviewed.

The 2011 Europhysics Conference on High Energy Physics-HEP 2011,

July 21-27, 2011

Grenoble, Rhône-Alpes France

\footnotetext{
* Speaker.

†nvited Talk
} 


\section{Introduction}

Since their discovery neutrinos have been studied extensively and the knowledge about their properties has advanced our understanding of weak interactions significantly. Still unanswered, however, is the very fundamental question whether the neutrino is a Majorana particle like most extensions of the Standard Model assume. While neutrino oscillation experiments can only provide data on the mass differences of the neutrino mass eigenstates, the neutrino absolute mass scale can only be obtained from direct measurements: the ${ }^{3} \mathrm{H}$ or other nuclei endpoint measurements or, in case of Majorana neutrinos, by neutrinoless double beta decay (DBD) experiments.

Neutrinoless $\operatorname{DBD}(0 v \beta \beta)$ is a very slow lepton-number violating nuclear transition that happens if neutrinos have mass and are their own antiparticles. An initial nucleus $(Z, A)$ decays to $(Z+2, A)$, emitting two electrons. A related transition, called two-neutrino DBD $(2 v \beta \beta)$ results in the emission of two electron anti neutrinos in addition to the charged leptons.

Many experiments, focusing on different candidate decay nuclides and exploiting various experimental techniques have been performed and searched for this rare decay. The most sensitive experiments performed so far on DBD have tested the rare decay ${ }^{76} \mathrm{Ge} \rightarrow{ }^{76} \mathrm{Se}+2 e^{-}$using Germanium detectors operated at cryogenic temperatures in a low background environment.

The best half-life limits come from the Heidelberg-Moscow [1] and IGEX [2] experiments with lower bounds on the ${ }^{76} \mathrm{Ge}$ half-life of $1.9 \cdot 10^{25} \mathrm{y}$ and $1.6 \cdot 10^{25} \mathrm{y}$, respectively. Other isotopes have been measured and the best lower bound for ${ }^{130} \mathrm{Te}$ is $2.8 \cdot 10^{24} \mathrm{y}[4]$ while for ${ }^{116} \mathrm{Cd}$ is 1.2 . $10^{19} \mathrm{y}$.

Evidence of $0 v \beta \beta$ in ${ }^{76} \mathrm{Ge}$ has been reported [3] but it has not been confirmed so far by other experiments.

For a generic DBD experiment, the half-life sensitivity for discovery at a given confidence level when the background rate is different from zero, can be expressed as

$$
T_{1 / 2}^{0 \nu} \propto \frac{\varepsilon a}{W} \sqrt{\frac{M T}{b \Delta E}},
$$

where $\varepsilon$ is the detection efficiency, $a$ the abundance of the DBD active isotope in the detector, $W$ the molecular weight of the source material, $M$ the source mass in $\mathrm{kg}, T$ the exposure time in years and $\Delta E$ the energy window of observation for the $0 v \beta \beta^{1}$ in units of $\mathrm{keV}$, and $b$ the specific background index in units of $\mathrm{keV}^{-1} \mathrm{~kg}^{-1} \mathrm{y}^{-1}$.

The present is a short report on the status of a selection of crystal based detectors: bolometers (CUORE), germanium diodes (GERDA and MAJORANA) and room temperature pixelated CdZnTe sensors (COBRA).

\section{The CUORE experiment}

The CUORE experiment, currently under construction at the Gran Sasso Underground Laboratories (LNGS), will be made of $988 \mathrm{TeO}_{2}$ detectors for a total mass of $\sim 206 \mathrm{~kg}$ of ${ }^{130} \mathrm{Te}$. CUORE will exploit the technology and experience gained with the CUORICINO [4] experiment which ran from 2003 to 2008 at LNGS. The background index in the region of interest has been

\footnotetext{
${ }^{1} \Delta E$ is related to the energy resolution at the $Q_{\beta \beta}$
} 
measured as $b=0.169 \pm 0.006$ counts/(keV kg y). Data, collected for an exposure of $19.6 \mathrm{~kg} \cdot \mathrm{y}$, allowed to set a lower limit on the half-life of $0 v \beta \beta$ decay of ${ }^{130} \mathrm{Te}, T_{1 / 2}>2.8 \times 10^{24} \mathrm{y}$ [4].

In CUORE, the bolometers will be operated in a special cryostat realized almost completely in copper which will cool down the detectors to $10 \mathrm{mK}$ by means of pulse tubes and by a high power ${ }^{3} \mathrm{He} /{ }^{4} \mathrm{He}$ dilution refrigerator. The cryostat, made of six vessels with two vacuum chambers, has a very compact structure and will allow to place the bolometers in a compact array allowing to reduce background by means of detectors anti-coincidence.

The first assembled tower, CUORE-0, is being prepared at LNGS and it will start operation by the end of the year in the refurbished CUORICINO cryostat.

Recent calculations on the CUORE sensitivity to DBD can be found here [5].

\section{The GERDA and Majorana experiments}

The GERDA experiment [6] at LNGS has been built to detect $0 v \beta \beta$ using Germanium diodes enriched in the ${ }^{76} \mathrm{Ge}$ isotope. The GERDA apparatus is made of a $65 \mathrm{~m}^{3}$ stainless steel cryostat with internal $\mathrm{Cu}$ shielding layers and filled with liquid Argon (LAr). The cryostat is placed inside a tank filled with $590 \mathrm{~m}^{3}$ of pure water. The LAr and water act as shielding materials against external background radiations (gammas and neutrons). The shielding against cosmic muons is given by the natural shielding of the Gran Sasso rocks (3800 m water equivalent) and by the active shield provided by the instrumented water volume around the cryostat: Cherenkov radiation produced by muons traversing the water tank is detected by 66 photo-tubes, and an additional veto is provided by plastic scintillator panels mounted on top of the experiment.

The GERDA physics program is organized in two steps. In Phase I, low background coaxial germanium detectors enriched in the ${ }^{76} \mathrm{Ge}$ isotope from the former Heidelberg-Moscow and IGEX collaborations are used. The total mass is about $18 \mathrm{~kg}$ and the design background index is $10^{-2}$ counts $/(\mathrm{keV} \cdot \mathrm{kg} \cdot \mathrm{y})$. For Phase II, new diodes made of ${ }^{76} \mathrm{Ge}$ enriched material will be built by Canberra using the BEGe technology [7]. This type of detectors has demonstrated to have excellent energy resolution and capabilities of discriminating between $0 v \beta \beta$ (single site) and $\gamma$ Compton scattering (multiple site) events [8]. For phase II new detectors with a total mass of $20 \mathrm{~kg}$ will be produced and the background index will be reduced to $10^{-3}$ counts $/(\mathrm{keV} \cdot \mathrm{kg} \cdot \mathrm{y})$.

The GERDA Collaboration started the commissioning of the apparatus in June 2010 using low background natural germanium detectors. The measurements have allowed to study, for the first time, the background of the full apparatus and to prepare the setup for the deployment of the enriched detectors and start the physics Phase I. The operation of natural germanium detectors ended in April 2011 and was followed by the installation of a test string with enriched detectors. The commissioning phase has been recently completed and at the beginning of November 2011, Phase I started.

The first operation of naked detectors in LAr showed a background contribution dominated by a line at $1525 \mathrm{keV}$ (from ${ }^{42} \mathrm{~K}$, a progeny of ${ }^{42} \mathrm{Ar}$ ). The measurement, performed with different biasing schemes gave evidence for charged potassium ions drifting in the electric fields surrounding the diodes ${ }^{2}$. For the following runs a mini-shroud (a cylinder surrounding the detectors in the string

\footnotetext{
${ }^{2}$ The $Q$ value of the $\beta$ decay of ${ }^{42} \mathrm{~K}$ is $3.5 \mathrm{MeV}$ and therefore a contribution at $Q_{\beta \beta}$ is expected.
} 


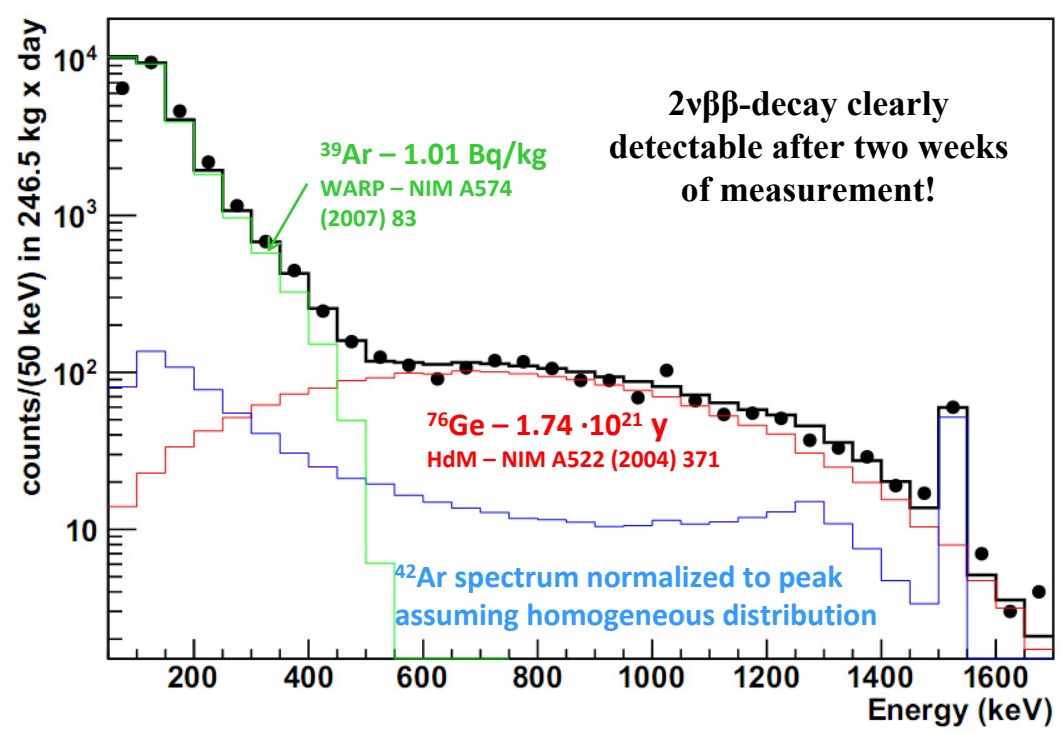

Figure 1: GERDA low energy spectrum measured with a string of enriched HPGe detectors. From[10].

made of $0.1 \mathrm{~mm}$ thick $\mathrm{Cu}$ foil) was used in order to reduce the LAr volume around the detectors from which the ions can be collected. The mini-shroud, electrically isolated from the holders, could be biased. Several runs with different electrical configurations were carried out.

The GERDA Collaboration has recently reported [10] on the first measurement of the background index using a string with three enriched germanium detectors (total mass of $6.7 \mathrm{~kg}$ ). Fig. 1 shows the energy spectrum of one enriched detector string. The spectrum (dots) is compared to Monte Carlo samples of the ${ }^{39} \mathrm{Ar}$ background $(\mathrm{E}<500 \mathrm{keV}),{ }^{42} \mathrm{Ar}$ and $2 v \beta \beta$ decay in the lower energy region. The ${ }^{39} \mathrm{Ar}$ and $2 v \beta \beta$ spectra were normalized to the Warp [11] and Heidelberg-Moscow [3] published results, while the ${ }^{42} \mathrm{Ar}$ is normalized to the observed data at the $1525 \mathrm{keV}$ peak. The measured background index, with a $100 \mathrm{keV}$ and $200 \mathrm{keV}$ energy window around the $Q_{\beta \beta}$ value is:

$$
b_{100 \mathrm{keV}}=0.017_{-0.012}^{+0.029} \mathrm{cts} /(\mathrm{keV} \mathrm{kg} \mathrm{y}) \quad b_{200 \mathrm{keV}}=0.035_{-0.015}^{+0.021} \mathrm{cts} /(\mathrm{keV} \mathrm{kg} \mathrm{y}) .
$$

While GERDA is a European experiment, the MAJORANA Collaboration is working on a similar project in the USA. The MAJORANA Demonstrator (MJD) [12] is a project testing the feasibility of building and operating an ultra-low background tonne-scale ${ }^{76} \mathrm{Ge} 0 v \beta \beta$ experiment. The project is organized in steps. The first phase will involve building two or three cryostats containing about $40 \mathrm{~kg}$ of Germanium detectors (with up to $30 \mathrm{~kg}$ of enriched diodes). The cryostats will be surrounded by a $4 \pi$ muon shielding and active veto. The background reduction is achieved on one side with a careful selection, screening and fabrication of detector materials for radiopurity, and on the other side with effective pulse-shape analysis of the detector signals. The latter is possible and very effective thanks to the BEGe detector technology, used also for GERDA Phase II. The MJD will be located at the 4850' level of Sanford Underground Lab at the Homestake mine in South Dakota. The operation of enriched germanium diodes will start in 2013. 
The GERDA and MAJORANA Collaborations intend to eventually join their efforts and come together to construct a tonne-scale experiment.

\section{The COBRA experiment}

The aim of the COBRA experiment [13] is to search for DBD using a large array of Cadmium Zinc Telluride $(\mathrm{CdZnTe})$ semiconductors operated at room temperature. One of the isotope of interest is ${ }^{116} \mathrm{Cd}$ were the $\mathrm{Q}_{\beta \beta}$ is above all single gamma lines from natural decay chains $(2.8$ $\mathrm{MeV})$. The development of pixelated detectors array provide an efficient background suppression: the combination of tracking information with energy deposition could be used to separate a DBD event (single site) from alpha particles, muons or single electrons events. The COBRA experiment is still in the $\mathrm{R} \& \mathrm{D}$ phase but the proposed approach looks very promising.

\section{References}

[1] H. V. Klapdor-Kleingrothaus, et al., Eur. Phys. J A12 (2001) 147-154.

[2] C. E. Aalseth, et al. IGEX Collaboration, Phys. Rev. D65 (2002) 092007.

[3] H. V. Klapdor-Kleingrothaus, et al., Mod. Phys. Lett. A16 (2001) 2409-2420.

H. V. Klapdor-Kleingrothaus, et al. Nucl. Instrum. Meth. A522 (2004) 371-406.

H. V. Klapdor-Kleingrothaus and I. V. Krivosheina, Mod. Phys. Lett. A21 (2006) 1547-1566.

[4] E. Andreotti et al, CUORICINO Collaboration, Astropart. Phys. 34 (2011) 822-831.

[5] F. Alessandria, et al., submitted to Astropart. Phys, nucl-ph 1109.0494v2

[6] I. Abt, et al., "GERDA The GERmanium Detector Array for the search of neutrinoless $\beta \beta$ decays

${ }^{76}$ Ge at LNGS", LNGS P38/04 Sept 2004.

[7] Broad Energy Germanium (BEGe), product of Canberra an AREVA Company, http://www.canberra.com

[8] GERDA Collaboration, "Isotopically modified Ge detectors for GERDA: full test from production to operation", in preparation.

[9] GERDA Collaboration, Reports to the LNGS Scientific Committee, LNGS-EXP 33/05, add: 11/10 (October), 12/11 (April 2011) and 13/11 (October 2011).

[10] B. Majorovits, International Workshop on Double Beta Decays and Neutrinos, November 14-17 2011, Osaka, Japan.

[11] P. Benetti, et al, Nucl. Instrum. Meth. A574 (2007) 83-88.

[12] E. Aguayo et al., DPF-2011 Conference, August 8-13, 2011, Providence, Rhode Island, USA. R. Martin et al., International Workshop on Double Beta Decays and Neutrinos, November 14-17 2011, Osaka, Japan.

[13] M. Schwenke, et al., Nucl. Instrum. Meth. A650 (2011) 73-78. 